\title{
Modeling Of Species Interaction in a Habitat Using Lotka- Volterra Type Systems
}

\author{
Nweze N. O. ${ }^{1}$, Offiong N. M. ${ }^{1 *}$, Adehi M. U. ${ }^{1}$, Chaku S. E. ${ }^{1}$, Abdullahi S. \\ A. ${ }^{1}$, Muhammad N. Mahammmad ${ }^{2}$ \\ ${ }^{1,2}$ Department of Mathematical Sciences, Nasarawa State University, Keffi, Nasarawa, Nigeria. \\ Correspondence: nonweze@yahoo.com \\ offiong.mitchel@nsuk.edu.ng
}

\begin{abstract}
Mathematical models have been useful in the area of modeling of real life situations; its application can be found in virtually all spheres of scientific researches. As such, we adopt its use in the field of ecology where preys have to compete with other prey for survival. In this paper, we considered Lotka-Volterra type systems, consisting of two first order differential equations which were used to model the population size of prey-predator interaction. We also proposed a system of first order differential equations to model the population sizes of a prey and two predators. Under these conditions one of the predators dies out while the remaining predator and prey approach periodic behavior as time increases. Also we model the population size of two preys and one predator where there may be interaction between the preys. Under these conditions we found that one of the preys died out while the remaining preys and predators approached periodic behavior as time increased. For critical cases, each positive solution of the system was seen to be periodic in nature. Various examples and results were presented and further study was proposed.
\end{abstract}

Keywords: Mathematical Models, Lotka-Volterra, differential equation, habitat, predator, prey.

\section{Introduction}

Ecologists strive to understand how interspecies interaction contributes to distribution and abundance of particular species. Decades of modeling, observation studies and experimental approaches have led ecologists to conclude that species sharing the same habitat often play important but highly diverse roles in affecting each other according to Chesson [1]. Keddy [2], mentioned that interactions among species were viewed as a simple mutually negative interaction either directly through interferences or indirectly through exploitation of shared resources. Understanding such mutually negative interactions among competing species remains a cornerstone of community ecology. Keddy showed apparent competition occurs when two or more prey species share a common predator, and predator's numbers are limited by prey availability. By presenting itself as an additional food resource, the second prey species allows the abundance of the predator to increase and, as a result reduces the density of the target species. If more than one prey species is present, and both utilities those enemy-free resources, the species can limit each other's numbers.

An apparent mutualism between prey species that share a common predator can arise if predator populations are limited by factors other than prey availability, Abrams [3]. In this case, the presence of a prey species satisfies the predator, thus allowing a relief of predator pressure on the target species; that is, the species indirectly benefits the target species. Co-operation or symbiosis infection occurs when two species help each other in some ways. Week-strong interaction occurs when one species is simply better suited to survive than other. Competition between species may be indirect, in which the competitors use a resource but do not confront each other over it, in direct competition, the two species may fight over a resource. Sometimes competition may be mediated by a third organism, such as predator or parasite. The Difference in size between a predator and its prey has an effect on the development of specialized structures of the predator for subduing the prey. For the most part, predator feed in species smaller than themselves; consider herbivorous to be predators of plants, just as carnivores are predators of animals.

\section{Literature Survey}

The Lotka-Volterra predator-prey and competition model was initially proposed by Alfred .J. Lotka in the theory autocatalytic chemical reaction in 1910. This was effectively the logistic equation, Berryman (1992), which was originally derived by Pierre Francis Verhulst. The Lotka-Volterra model and Holling's extension have been used to model the moose and wolf population Jost[4].

Given two species of animals, interdependence might arise because one species (the prey) serves as food source for the other species (the predator). Models of this type are thus called predator prey models. Let $\mathrm{P}$ 
denote the size of the prey population and $\mathrm{Q}$ donate the size of the predator population. The growth rate of prey populations is determined by the equation:

$$
\frac{d P}{d t}=\alpha\left(1-\frac{P}{Q}\right)-\beta Q
$$

where $\alpha, \beta$ and $Q$ are parameters, Barntt [5]. In the absence of predators (when $Q=0$ ) the growth of the prey population thus follows the logistic model where $Q$ is called carrying capacity of the environment. The growth rate of the predator is determined by the equation.

$$
\frac{d Q}{d t}=-\gamma+\delta P Q \lambda
$$

where $\gamma, \delta$ are parameters. In the absence of prey (when $P=0$ ) the predator population would shrink at rate $\gamma$. However, the predator growth rate rises as the prey population becomes larger. The two equations (1) and (2) can be described as:

$$
\begin{aligned}
& \frac{d P}{d t}=\left(\alpha\left(1-\frac{P}{K}\right)-\beta Q\right) P h . \\
& \frac{d Q}{d t}=(-\gamma+\delta P) Q h \ldots \ldots \ldots \ldots . . .
\end{aligned}
$$

where $\mathbf{h}$ is the period of the interaction, this version of the predator prey model provides a useful starting point, provides the needed basic insight that more predators are bad for prey, while more prey are good for predators. In the Lotka-Volterra model, the prey population faces a capacity constraint giving by parameter $K$, if there is no capacity constraint so that $\mathrm{k}$ is infinite the two equation system is giving below.

In the development of this model, a number of assumption, were made by Lotka-Volterra. These include:

- If there are no predators, the prey population will grow at the rate proportional to the population of the prey species.

- If there are no prey, the species decline at the rate proportional to the population if the predator.

The presence of both predators and prey is beneficial to growth of predators' species and is harmful to growth of prey species. More especially the predator species increases and the prey species decreases at rate proportional to the product of the two populations.

During the process, the environment does not change in favor of one species and the genetic adaptation is sufficiently slow. As differential equations are used, the solution is deterministic and continues. This in turn, implies that the generations of both the predators and prey are continually over lapping Cook [6]. This assumption gives the system of non-linear first order ordinary differential equations.

$$
\begin{gathered}
\frac{d p}{d t}=p(\alpha-\beta Q) \\
\frac{d Q}{d t}=-Q(\gamma-\delta p) .
\end{gathered}
$$

Where:

$$
\begin{aligned}
& P=\text { prey population } \\
& Q=\text { predator population } \\
& \alpha=\text { the growth rate of prey } \\
& \gamma=\text { death rate of predator } \\
& \delta=\text { growth rate of predator }
\end{aligned}
$$

Equation (5) can be express as:

$$
\frac{d p}{d t}=p(\alpha-\beta Q)
$$

This means the change in prey's numbers is given by its own growth minus the rate at which it is preyed upon. Equation (6) can also be express as:

$$
\frac{d Q}{d t}=-\gamma Q+\delta P Q
$$

The equation expresses the change in the predator population as the death rate of predator plus growth rate of the predator.

The above equations (7) and (8) can be solved numerically and the orbits can be defined as follows: 


$$
\begin{gathered}
\frac{d Q}{d P}=\frac{-Q(\gamma-\delta P)}{P(\alpha-\beta Q)} \\
\frac{d Q}{d P}=\frac{-Q \gamma+\delta P Q}{P \alpha-\beta P Q} \\
\frac{d Q}{d P}=\frac{\delta\left(-\frac{\gamma}{\delta}+P\right) Q}{\beta\left(\frac{\alpha}{\beta}-Q\right) P}
\end{gathered}
$$

Equation (9) is a separable first order differential equation and may be written as:

$$
\begin{gathered}
\frac{\alpha-\beta Q}{Q} \cdot \frac{d Q}{d t}=\frac{-\gamma+\delta P}{P} \cdot \frac{d P}{d t} \\
\left(\frac{\alpha}{Q}-\beta\right) \frac{d Q}{d t}=\left(\frac{-\gamma}{P}+\delta P\right) \frac{d P}{d t}
\end{gathered}
$$

Integrating equation (10) yields

$$
\alpha \operatorname{In} Q-\beta Q=(-\gamma \operatorname{In} P+\delta P)+C
$$

From equation (11), we have that

$$
\begin{aligned}
& Q^{\alpha} \ell^{-\beta Q}=C P-\gamma \ell^{\delta P} \\
& F(P Q)=\left[\frac{P^{\gamma}}{\ell^{\delta P}}\right]\left[\frac{Q^{\alpha}}{\ell^{\beta Q}}\right]=C \\
& \propto, \beta, \Upsilon, \delta>0
\end{aligned}
$$

This gives the $F(P Q)=\mathrm{C}$ described in fig. (2-1)

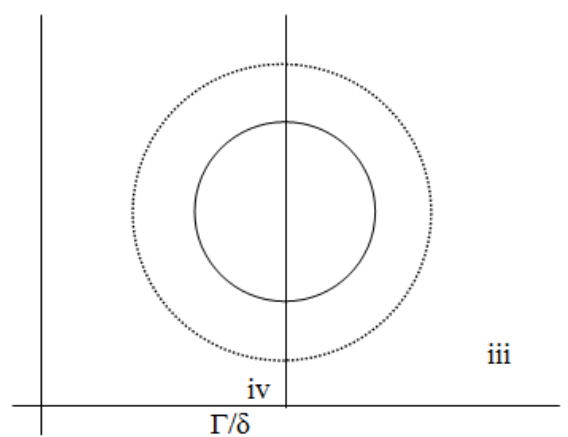

Fig. 1: Decision Quadrant

The lines through $(\gamma / \beta, \gamma / \delta)$ parallel to the axes of co-ordinates divides the first quadrant into four parts $\mathrm{i}$, ii, iii and iv using equation (5) and (6), we found the following behaviors
In $\quad \mathrm{i} \quad \frac{d P}{d t}<0, \quad \frac{d Q}{d t}>0$
In $\quad$ ii $\quad \frac{d P}{d t}<0, \quad \frac{d Q}{d t}<0$
In $\quad$ iii $\quad \frac{d P}{d t}>0, \quad \frac{d Q}{d t}<0$
In $\quad$ iv $\quad \frac{d P}{d t}>0, \quad \frac{d Q}{d t}>0$ 


\section{Model Assumption:}

\section{Method}

In an actual environment, there are a number of different predator-prey relationships that could single prey species this scenario is examined, and the predator-prey interaction dynamics model can be expressed as;

$\frac{d X}{d t}=a X-b X Y-g X Z$

$\frac{d Y}{d t}=-d Y+c X Y$

$\frac{d Z}{d t}=-f Z+e X Z$

\section{Dynamics of the Model}

In the model system, the predators thrive when there is plentiful prey but ultimately, outstrip their food supply and decline. If the predator population is low, then the prey population will increase again.

\section{Population Equilibrium}

Population equilibrium occurs in the model when neither of the population levels is changing. When $\frac{d X}{d t}=0, \frac{d Y}{d t}=0, \frac{d Z}{d t}=0$.

From equation (3.1) we have:

$$
\begin{aligned}
& a X-b X Y-g X Z=0 \\
& X(a-b Y-g Z)=0 \\
& X=0 \\
& a-b Y-g Z=0 \\
& g Z=a-b Y \\
& Z=\frac{a-b Y}{g}
\end{aligned}
$$

Also

$$
\begin{aligned}
& b Y=a-g Z \\
& Y=\frac{a-g Z}{b}
\end{aligned}
$$

From equation (3.2) we have,

$$
\begin{aligned}
& Y(-d+c X)=0 \\
& Y=0 \\
& -d+c X=0 \\
& X=\frac{d}{c}
\end{aligned}
$$


Also, from equation (3.3) we have,

$$
\begin{aligned}
& Z(-f+e X)=0 \\
& Z=0,-f+e X=0 \\
& X=\frac{f}{e}
\end{aligned}
$$

There are three equilibriums

$$
(0,0,0),\left(\frac{d}{e}, 0, \frac{a-b Y}{g}\right) \text { and }\left(\frac{f}{e}, \frac{a-g Z}{B}, 0\right)
$$

The equilibrium point $\left(\frac{f}{e}, \frac{a-g Z}{g}, 0\right)$ exist if $\mathrm{f}>$ ed, the predator prey model in this case, we conclude that $\mathrm{Z}$ predator dies out i.e. fails to persist and $\mathrm{X}(\mathrm{t})$ and $\mathrm{Y}(\mathrm{t})$ are periodic. The equilibrium point $\left(\frac{d}{c}, 0, \frac{a-b Y}{g}\right)$ exist if $\mathrm{f}<\mathrm{ed}$, the predator prey model in this case, we conclude that $\mathrm{Y}$ predator fails to persist and $\mathrm{X}(\mathrm{t})$ and $\mathrm{Z}(\mathrm{t})$ are periodic.

\section{Stability of the fixed point}

The stability of the fixed point at the origin can be determined by using linearization.

The Jacobean of the two predator prey model is:

$$
J(X, Y, Z)=\left(\begin{array}{ccc}
a-b Y-g Z & -b X & -g X \\
c Y & c X-d & 0 \\
e Z & 0 & e X-f
\end{array}\right)
$$

At the equilibrium point $(0,0,0)$ the Jacobean matrix becomes,

$$
J(0,0,0)=\left(\begin{array}{ccc}
a & 0 & 0 \\
0 & -d & 0 \\
0 & 0 & -f
\end{array}\right)
$$

The characteristic equation is

$$
\begin{aligned}
& a-y[(-d-\lambda)(-f-\lambda)]=0 \\
& a-\lambda=0,-d-\lambda=0,-f-\lambda=0 \\
& a=\lambda,-d=\lambda,-f=\lambda
\end{aligned}
$$

In the model $\mathrm{a}, \mathrm{d}$ and $\mathrm{f}$ are always $>0$ and as such the sign of the eigen-values will always differ. Hence the fixed point at the origin is unstable since one of the eigen-values is positive and the other negative. If it were stable, the dynamics of the system might lead towards the extinction of the species.

At the equilibrium point $\left(\frac{d}{c}, 0, \frac{a-b Y}{g}\right)$ the Jacobean matrix is 


$$
J\left(\frac{d}{c}, 0, \frac{a-b Y}{g}\right)=\left(\begin{array}{ccc}
a-\frac{g(a-b Y)}{g} & b\left(\frac{d}{c}\right) & g\left(\frac{d}{c}\right) \\
0 & c\left(\frac{d}{c}\right)-d & 0 \\
e\left(\frac{a-b Y}{g}\right) & 0 & e\left(\frac{d}{c}\right)-f
\end{array}\right)
$$

The characteristic equation is:

$$
\begin{aligned}
& b Y-\lambda[-\lambda(k-\lambda)]+i(j-\lambda)=0 \\
& -b Y-\lambda=0,-\lambda=0, k-\lambda=0 \\
& \lambda=b Y, \lambda=0, \lambda=k
\end{aligned}
$$

Where

$$
i=g\left(\frac{d}{c}\right), j=e\left(\frac{a-b Y}{g}\right) \text { and }, k=e\left(\frac{d}{c}\right)-f
$$

Since the eigen-values have different signs the equilibrium point is unstable.

At the equilibrium point $\left(\frac{f}{e}, \frac{a-g Z}{b}, 0\right)$ the Jacobean matrix is:

$J\left(\frac{f}{e}, \frac{a-g Z}{b}, 0\right)=\left(\begin{array}{ccc}a-\frac{b(a-g Z)}{b} & -b\left(\frac{f}{e}\right) & -g\left(\frac{f}{e}\right) \\ c\left(\frac{a-g Z}{b}\right) & c\left(\frac{f}{e}\right)-d & 0 \\ 0 & 0 & e\left(\frac{f}{e}\right)-f\end{array}\right)$

Since the eigen-values have different roots, the point is unstable.

The characteristics equation now becomes:

$$
g Z-\lambda[-\lambda(-\lambda)+i(j-\lambda)-w(-\lambda)]=0
$$

One of the eigenvalues is $\lambda=g Z$

The other two eigen-values are given by the roots of the following quadratic equation $\lambda^{2}+\lambda(-i+w)+i j=0$

\section{Definition of parameters and variables for the model equation II}
$\lambda_{1}, \lambda_{2}$
Are biotic potentials
$k_{1}, k_{2}$
Carrying capacities of the two prey species
$\alpha_{12}$
Effect species 2 has on the population of species 1
$\alpha_{21}$
Effect species 1 has on the population of species 2
$\alpha_{13}, \alpha_{23}$
Predator coefficients
$\alpha_{31}, \alpha_{32}$
Growth rate of predators 


$\begin{array}{ll}\alpha_{33} & \text { Death rate of the predator } \\ \mathrm{E} & \text { Harvesting effect } \\ \mathrm{q}_{1}, \mathrm{q}_{2} & \text { Catch ability coefficient of } \mathrm{X}_{1} \text { and } \mathrm{X}_{2} \text { respectively. } \\ \mathrm{X}_{1} & \text { First prey population } \\ \mathrm{X}_{2} & \text { Second prey population } \\ \mathrm{X}_{3} & \text { Predator population }\end{array}$

\section{Assumption of the model equation II}

The ecological system is as follows. When two fish species compete with each other for the same resources, there is a predator (for example, a whale) feeding on both of them, both of the preys are subjected to continuous harvesting. Thus the interaction between the harvesting agency and the predator is through the third party, namely the prey to increase linearly with prey density. The governing equations of the system can be written as:

$$
\begin{aligned}
& d x_{1} / d t=X_{1}\left[\lambda_{1}\left(1-x_{1} / k_{1}\right)-\alpha_{12} x_{2}-x_{13} x_{3}\right]-q E x_{1} \\
& d x_{2} / d t=X_{2}\left[\lambda_{2}\left(1-x_{2} / K_{2}\right)-\alpha_{21} x_{1}-\alpha_{23} x_{3}\right]-q_{2} E x_{2} \\
& d x / d t=x_{3}\left[\alpha_{31} x_{1}+\alpha_{32} x_{1}-\alpha_{33}\right]
\end{aligned}
$$

\section{Steady states}

The steady states of the system are $\mathrm{P}_{1}\left(0_{1}, 0_{1}, 0_{1}\right), P_{2}\left(\begin{array}{lll}0, & X_{2}, X_{3}\end{array}\right)$

And $\mathrm{P}\left(X_{1,} \quad 0, X_{3}\right)$

Where $\quad X_{2}=\frac{\lambda_{2}-q_{2} E}{\lambda_{2} / K_{2}=\alpha_{32} \alpha_{23}} \quad X_{3}=\frac{\alpha_{32}\left(\lambda_{2}-q_{2 E}\right)}{\lambda_{2} / K_{2}+\alpha_{32} \alpha_{23}}$

$$
X_{1}=\frac{\lambda_{1}-q_{1} E}{\lambda_{1} / k_{1}+\alpha_{31} \alpha_{13}} \quad X_{3}=\frac{\alpha_{31}\left(\lambda_{1}-q_{1} E\right)}{\lambda_{1} / k_{1}+\alpha_{31} \alpha_{13}}
$$

The equilibrium point $\mathrm{P}_{1}$ exist if $\mathrm{E}<\lambda_{2} / q_{2}$ and $P_{2}$ exist if $\mathrm{E}<\lambda_{1} / q_{1}$.

The ratio $\lambda / q$ of the biotic potential to the catch ability productivity of the species.

\section{Local stability}

We examined the model equation for stability at the steady state

Let,

$\mathrm{V}\left(\mathrm{X}_{1}, X_{2}, X_{3}\right)=\left(\begin{array}{ccc}V 11 & -\alpha_{21} x_{1} & -\alpha_{13} x_{3} \\ -\alpha_{21} x_{2} & V 22 & -\alpha_{23} x_{2} \\ \alpha_{31} x_{3} & \alpha_{32} x_{3} & \alpha_{13} x_{1}+\alpha_{32} x_{2}-\alpha_{33}\end{array}\right)$

Where $\mathrm{V} 11=\left(\lambda_{1}-\frac{2 \lambda_{1}}{k_{1}} x_{12}-\alpha_{12} x_{2}-\alpha_{13} x_{3}-q_{1} E\right)$ 
$\mathrm{V} 22=\left(\lambda_{2}-\frac{2 \lambda_{2}}{k_{2}} x_{2}-\alpha_{21} x-\alpha_{23} x_{3}-q_{2} E\right)$

At the steady $(0,0,0)$, we have,

$\mathrm{V}(0,0,0)=\left(\begin{array}{ccc}\lambda_{1}-q_{1} E & 0 & 0 \\ 0 & \lambda_{2}-q_{2} E & 0 \\ 0 & 0 & 0\end{array}\right)$

The eigen-values of this matrix are $0, \lambda_{1}-q_{1} E, \lambda_{2}-q_{2} E$

$\mathrm{v}\left(0, X_{2}, X_{3}\right)=\left(\begin{array}{ccc}\lambda_{1}-\alpha_{12} x-\alpha_{13} x_{3}-q_{2} E & 0 & 0 \\ -\alpha_{21} x_{2} & -\lambda_{2} X_{2} / k_{2} & -\alpha_{23} x_{2} \\ \alpha_{31} x_{3} & \alpha_{32} x_{3} & x_{3}\end{array}\right)$

The characteristic equation is:

$\left[\left(\lambda_{1}-\alpha_{12} x_{2}-\alpha_{13} x_{3}-q_{1} E\right)-\eta\right] \eta^{2}+\eta\left(\frac{\lambda_{2}}{k_{2}} x_{2}+x_{3}\right)+\left(\frac{\lambda_{2}}{k_{2}} x_{2} x_{3}+\alpha_{23} \alpha_{32} x_{2} x_{3}\right)=0$

One of the eigenvalues of the matrix $\mathrm{V}\left(0, X_{2}, X_{3}\right)$ is

$\lambda_{1}-\alpha_{12} x_{2}-\alpha_{13} x_{3}-q_{1} E$.

The other two eigenvalues are given by the roots of the following quadratic equation

$\eta^{2}+\eta\left(\lambda_{2} x_{2} / k_{2}+x_{3}\right)+\left(\frac{\lambda_{2}}{k_{2}}+\alpha_{23} \alpha_{32}\right) x_{2} x_{3}=0$

In equation (3.4) the roots $=-\left(\lambda_{2} x_{2} / k_{2}+x_{3}\right)$ and product of the roots $=\left(\lambda_{2} / k_{2}+\alpha_{23} \alpha_{32}\right) x_{2} x_{3}$

Therefore the roots are real and negative or complex conjugates having negative real parts.

Hence it is unstable.

$$
v\left(X_{1}, 0, X_{3}\right)=\left(\begin{array}{ccc}
-\lambda_{1} x_{1} k_{1} & -\alpha_{21} x_{1} & -\alpha_{13} x_{1} \\
0 & \lambda_{2}-\alpha_{21} x_{1}-\alpha_{23} x_{3}-q_{2} E & 0 \\
\alpha_{31} x_{3} & \alpha_{32} x_{3} & -x_{3}
\end{array}\right)
$$

One of the eigen-values of the matrix is

$\lambda_{2}-\alpha_{21} x_{1}-\alpha_{23} x_{3}-q_{2} E$

The other two eigen-values are given by the roots of the quadratic equation

$\eta^{2}+\eta\left(\lambda_{1} x_{1} / k_{1}+x_{2}\right)+\left(\lambda_{2} / k_{2}+\alpha_{31} \alpha_{13}\right) x_{1} x_{2}=0$

In equation (3.5) the sum of the roots $=-\left(\lambda_{1} x_{1} / k_{1}+x_{2}\right)$ and product of the roots $=$

$\left(\lambda_{2} / k_{2}+\alpha_{31} \alpha_{13}\right) x_{1} x_{2}$

Therefore the roots are real and negative. Thus the equilibrium point $\left(\mathrm{X}_{1}, 0, X_{3}\right)$ is not stable.

\section{Conclusion}

A number of different situations may arise in a two predator and prey relationship, specifically two main conditions. Primarily, the three populations may be cyclical, as depicted in figure (2) below. We simulated the model equation (3.1), (3.2) and (3.3) using the following constants: 
$\mathrm{a}=2, \mathrm{~b}=2, \mathrm{c}=2, \mathrm{~d}=1, \mathrm{e}=1, \mathrm{f}=1 / 2 \mathrm{~g}=1$

$\mathrm{X}$ (prey population) is red, $\mathrm{Y}$ (first predator population) is blue, $\mathrm{Z}$ (second predator population) is green.

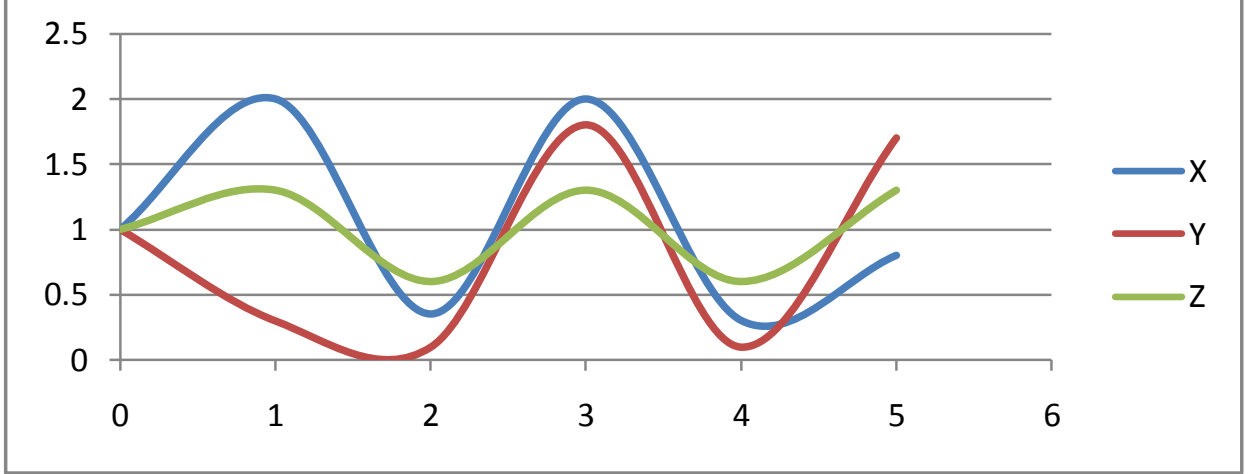

Fig. 2: Predator Population Chart

Secondly, since both predators derive their sole nourishment from the same prey species, the most efficient predator will eventually dominate the relationship and grow at a vast rate, whereas, the other predator will dwindle in number. The efficient predator and prey relationship shows a continuously cyclical pattern as shown in fig 4.2 below. The graph clearly illustrates in that in each cycle the prey population is reduced to extremely low number, but yet recovers while the predator population remain sizeable at the lowest prey density.

Constants $\mathrm{a}=2, \mathrm{~b}=1, \mathrm{c}=1, \mathrm{~d}=1, \mathrm{e}=1, \mathrm{f}=0.5, \mathrm{~g}=1$.

$\mathrm{X}$ (prey population) is red, $\mathrm{Y}$ (first predator population) is blue and $\mathrm{Z}$ (second predator population) is green.

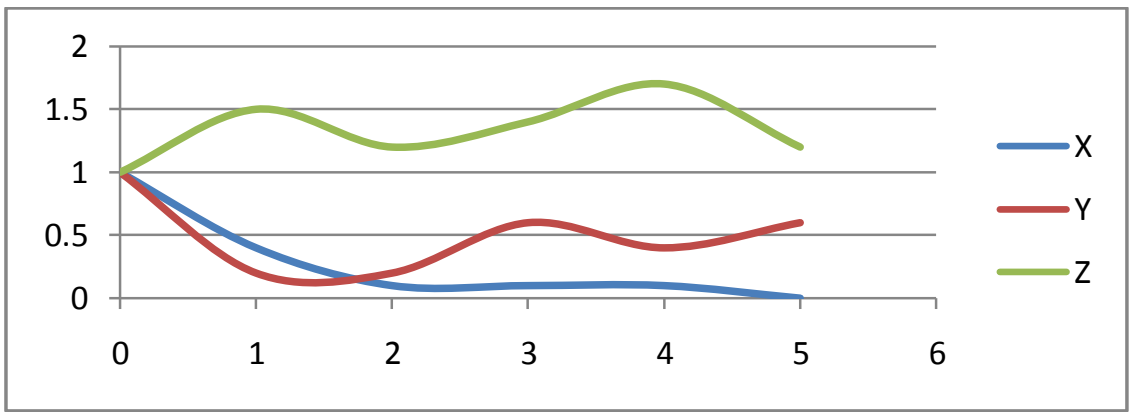

Fig. 3. Predator Population II

If the contact rate is reduced, then the prey population will increase and the predator population will reduce as shown in fig 4.3 below.

Constants $\mathrm{a}=2, \mathrm{~b}=0.5, \mathrm{c}=0.5, \mathrm{~d}=0.5, \mathrm{e}=0.5, \mathrm{f}=1, \mathrm{~g}=0.5$

$\mathrm{X}$ (prey population) is red, $\mathrm{Y}$ (first predator population) is blue and $\mathrm{Z}$ (second predator population) is green.

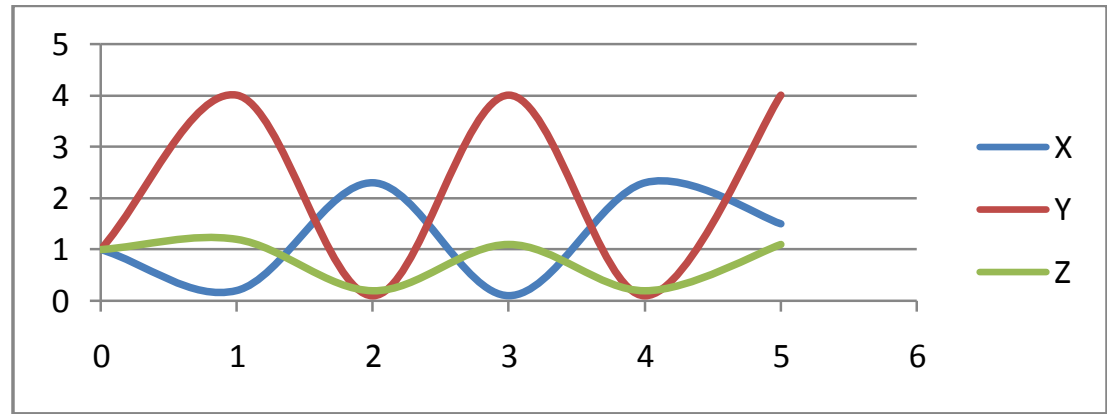

Fig 4: Prey Population

However, unlike the model where two predators hunted a single prey population, there is only a single situation that may arise regarding to one-to-two predation. As the single predator has two alternate food sources, the predator is able to hunt one of the prey populations to extinction with no consequential result. The predator population will then enter a continuous, cyclical population growth relationship with remaining prey as demonstrated in fig 4 below. 
Let

$$
\begin{aligned}
& \lambda_{1}=2.09, \lambda_{2}=2.07, k_{1}=200, k_{2}=300, q_{1}=0.04, q_{2}=0.01, \alpha_{12}=0.001, \alpha_{13}=0.01, \alpha_{31}=0.30, \\
& \alpha_{23}=0.02, \alpha_{32}=0.30
\end{aligned}
$$

$\mathrm{X} 1$ (first prey population) is blue, $\mathrm{X} 2$ (second prey population) is red and $\mathrm{X} 3$ (predator population) is green.

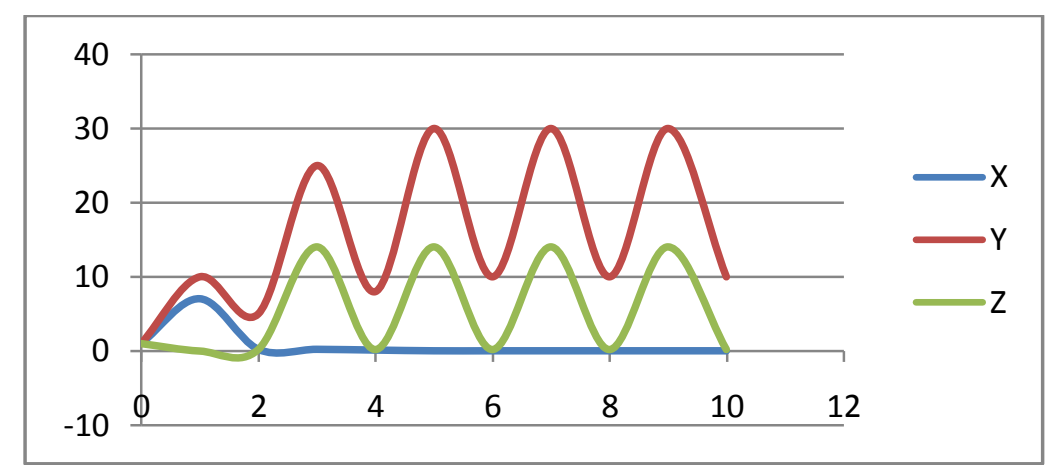

The stability of the steady state is of important since the eigen-valves have different roots, in general it is unstable. If it were stable, none zero population might be attracted towards the extinction of both species for many cases of initial levels. However as the fixed point at the origin is unstable, we find that the extinction of the species is difficult in all the models. This can only occur if the prey species are completely eradicated causing the predators to die of starvation. If the predators are completely eradicated the prey population grows without bound in these model.

In actual environmental settings, there are vast amount of complex, intermingled relationships between predators and prey. There is rarely a simple one predator population that only predates on a single prey population. In this paper, we have attempted to model the population size of two prey species competitive system in the presence of the predator species. Also one prey species and two predator's species. We have first studied the existence and stability of the possible steady states. In general there is no stable state.

\section{References}

[1] Chesson p., (2002), the interaction between predation and competition: a review and synthesis. Ecol. Lett. 5: 302-15

[2] $\operatorname{Keddy}(2001)$, Competition. Dordrcht: kluwer, $2^{\text {nd }}$ edition.

[3] Abrams P. A., (2001), Positive indirect effects between prey species that share predators.

[4] Lokta A. J., (1924), Elements of physical biolog Williams \& Wilkins, Baltimore.

[5] Barntt V. D., (1962), the monte carlo solution of a competing species problem.

[6] Cooke D., (1996), the mathematical theory of the dynamics of biological populations II, academic press Inc. 